\title{
educação

\section{O autoconceito de estudantes de ensino médio e sua relação com desempenho acadêmico: Uma revisão sistemática}

\author{
Guilherme da Silva Gasparotto ${ }^{\mathrm{I}}$ \\ Universidade Federal do Paraná \& Instituto Federal de Educação, \\ Ciência e Tecnologia (IFPR), Brasil
}

\section{Thaynara do Prado Szeremeta ${ }^{\mathrm{II}}$, Gislaine Cristina Vagetti ${ }^{\mathrm{II}}$, TAnia Stoltz ${ }^{\mathrm{IV}} \&$ VAldomiro de Oliveira $^{\mathrm{V}}$ Universidade Federal do Paraná, Brasil}

\begin{abstract}
O objetivo do estudo foi revisar sistematicamente as pesquisas que analisaram a relação do autoconceito com o rendimento acadêmico de adolescentes do ensino médio, bem como verificar quais variáveis e contextos dentro da escola foram abordados em conjunto ao autoconceito, para melhor explicar essa relação. A revisão incluiu estudos publicados nos últimos 10 anos nas bases eletrônicas de trabalhos científicos: ERIC/Thesaurus, MEDLINE/ Pubmed, SportDiscus, SciELO e Web of Science. Foram utilizados os descritores caracterizando componentes do autoconceito (self, self-concept e self-psychology) com os descritores relacionados ao rendimento acadêmico, em que também se considerou a capacidade acadêmica percebida (academic achievement, underachievement, educational achievement, perception of competence, competence perception, self-efficacy). Dez estudos foram incluídos na revisão. A relação entre autoconceito e desempenho acadêmico mostrou-se consistente e se confirmou em nove dos 10 trabalhos. Outras variáveis também se apresentaram importantes na explicação do desfecho do rendimento acadêmico, tais como a motivação acadêmica, a prática de atividades extracurriculares, a autoeficácia acadêmica e o envolvimento dos pais.
\end{abstract}

Palavras-chave: Estudantes; Psicologia do self; Adolescente; Avaliação do rendimento

\section{N T R O D U Ç Ã O}

O desempenho acadêmico é resultado da interação de diversos fatores intrínsecos e extrínsecos à pessoa (Allen, Gregory, Mikami, Lun, \& Hamre, 2013; Stankov, Lee, Luo, \& Hogan, 2012). O rendimento apresentado pelo estudante durante o processo de aprendizagem escolar depende de outros fatores, para além da exposição e interação com o conteúdo científico formal apresentado pela instituição. Sabe-se que a apreensão do conhecimento por parte do estudante pode estar relacionada, por exemplo, com as condições sociais, relações pessoais e 
condições psicológicas deste (Hines \& HolcombMcCoy, 2013).

Nessa perspectiva, a escola e seus agentes podem interferir, positiva ou negativamente, em alguns fatores, face a outros. Possivelmente, a instituição de ensino terá mais dificuldade em atuar sobre questões como a condição social do estudante ou relações pessoais externas à escola do que em aspectos psicológicos do estudante. Nesse sentido, os profissionais que atuam diretamente com o estudante podem ser determinantes na identificação e tomada de decisão quanto a fatores relacionados ao desenvolvimento psicológico, que podem afetar diretamente o seu rendimento acadêmico (Allen et al., 2013).

Estudos da área da psicologia da educação identificaram e discutem algumas variáveis que se relacionam com o desempenho acadêmico, há algumas décadas (Covington, 2000; Purkey, 1970). Entre esses trabalhos, o autoconceito é apresentado como uma importante variável, na qual os agentes escolares poderiam interferir, como forma de auxiliar no desenvolvimento do processo de aprendizagem do estudante (Klapp, 2017; Parker, Marsh, Ciarrochi, Marshall, \& Abduljabbar, 2014). Alguns autores citam, inclusive, que o autoconceito pode ser o principal construto afetivo-emocional relacionado com o sucesso acadêmico (Cia \& Barham, 2008).

$\mathrm{O}$ autoconceito pode ser definido como a percepção que o indivíduo tem de si próprio e o conceito formado dele mesmo, baseado no ideal projetado socialmente (Vaz Serra, 1988). Vaz-Serra (1998) cita que o autoconceito pode auxiliar a compreender aspectos importantes do comportamento humano. Esse construto pode ser percebido em algumas dimensões, como os autoconceitos acadêmico, emocional, social ou físico. Cada qual liga-se a situações diferentes do comportamento humano.

Com base em construtos como autoconceito e autoeficácia, pesquisas como a de Zimmerman
(2008) discutem os processos de autopercepções e crenças individuais, as quais favorecem ao estudante transformar competências psicológicas em competências de desempenho escolar. Competências psicológicas podem implicar o processo de aprendizagem em termos cognitivos, motivacionais e comportamentais. Essas autopercepções podem, então, segundo Zimmerman (2008), desenvolver o processo de autorregulação, que surge, portanto, como uma competência que faz o intermédio entre as competências psicológicas e a manifestação dessas capacidades em desempenho acadêmico.

No contexto acadêmico, uma percepção elevada de construtos como o autoconceito pode favorecer o desempenho, uma vez que se relaciona com a disposição do estudante em traçar metas e objetivos concretos, que, por sua vez, podem influenciar estratégias de estudo mais efetivas e elevação do rendimento (Valle et al. 2009). Além disso, algumas pesquisas revelaram que a estipulação de metas acadêmicas apresenta correlações positivas com a regulação do esforço nos estudos, a valorização das tarefas acadêmicas, a percepção de autoeficácia e a gestão do tempo e ambiente de estudo (Miranda \& Almeida, 2009; Valle et al., 2009).

Apesar de diversas pesquisas apresentarem relações importantes do autoconceito com o rendimento escolar, grande parte dos estudos que analisaram essas relações foram desenvolvidos com amostras de crianças. Diante disso, torna-se difícil expandir o entendimento desses resultados para outros grupos dentro da escola, em especial os adolescentes (Hattie, 2014). Cabe ressaltar que, embora alguns trabalhos com adolescentes sejam observados na literatura internacional, até ao presente não há registros de pesquisas que explorem a relação do autoconceito com o rendimento acadêmico de estudantes dessa faixa etária no Brasil. Dessa forma, um levantamento dessa natureza pode evidenciar a importância de se explorar o tema nacionalmente. 
Entender como se apresenta a relação do autoconceito com o desempenho acadêmico entre os adolescentes tem especial importância, uma vez que essa fase do desenvolvimento humano é marcada por alterações e definições de conceitos e comportamentos, que podem ser determinantes para um bom desenvolvimento psicológico e, consequentemente, para o controle e sucesso em diversas dimensões da vida, inclusive em relação ao ambiente escolar (Klapp, 2017). Além disso, é fundamental entender quais e como outras variáveis conjuntas ao autoconceito, bem como contextos diferentes dentro da escola, podem estar relacionados ao rendimento acadêmico do estudante adolescente. Com o conhecimento dos fatores que podem contribuir para a explicação do rendimento acadêmico, posteriormente será possível delinear estudos que sugiram abordagens pedagógicas favorecedoras de atributos psicológicos, tais como o autoconceito.

Diante do exposto, este trabalho tem como objetivo revisar sistematicamente os estudos que analisaram a relação do autoconceito com o rendimento acadêmico de adolescentes, especificamente estudantes do ensino médio, bem como verificar, nesses estudos, quais variáveis e contextos, dentro da escola, são abordados em conjunto com o autoconceito, para melhor explicar a relação com o rendimento acadêmico.

\section{MÉTODO}

\subsection{TIPO DE ESTUDO}

Trata-se de um estudo de revisão sistemática da literatura, que é um dos delineamentos mais adequados para se verificar o estado da arte sobre algum tema específico e sintetizar o conhecimento atual sobre determinada relação entre fatores ou variáveis. A partir da produção de uma revisão sistemática, se permite, além de aprofundar o conhecimento sobre a temática investigada, apontar lacunas que precisam ser preenchidas por meio da realização de novas investigações (Ribeiro, 2014).

O desenho metodológico de uma revisão sistemática tem alto desempenho na identificação de evidências científicas. Segundo o Oxford Centre for Evidence-Based Medicine (OCEBM, 2016), esse tipo de pesquisa está classificada como nível 1 de cinco possíveis, na representação dessas evidências, visto que possibilita estabelecer um panorama sobre o tema estudado.

\subsection{ESTRATÉGIA DE BUSCA}

A identificação dos artigos incluídos nesta revisão foi feita por meio de busca em cinco bases eletrônicas de dados: ERIC/Thesaurus, MEDLINE/Pubmed, SportDiscus, SciELO e Web of Science. A busca dos artigos foi limitada ao período entre 2007 e 2017, tendo-se considerado artigos publicados nos idiomas português, inglês e espanhol, visto que textos e periódicos de maior impacto acadêmico são publicados ou traduzidos para esses idiomas. Foram utilizados descritores caracterizando componentes do autoconceito (self, self-concept e self-psychology) e descritores relacionados ao rendimento acadêmico, em que também se considerou a capacidade acadêmica percebida (academic achievement, underachievement, educational achievement, perception of competence, competence perception, self-efficacy).

As buscas de estudos foram realizadas com descritores em língua inglesa e portuguesa. Foram realizadas combinações entre os descritores mediante a utilização dos operadores booleanos “AND” e "OR”. Não foram incluídas teses, dissertações e monografias. Todos os processos de seleção e avaliação de artigos foram realizados por pares.

Uma análise inicial foi realizada com base nos títulos dos manuscritos; em seguida, outra avaliação foi realizada nos resumos de todos os artigos que preenchiam os critérios de inclusão ou que não permitiam certeza de que deveriam 
ser excluídos. Após análise dos resumos, todos os artigos selecionados foram obtidos na íntegra e, posteriormente, examinados de acordo com os critérios de inclusão estabelecidos. Também foi realizada uma busca manual em listas de referências dos artigos selecionados, bem como os primeiros autores dos manuscritos foram contatados, visando a identificação de outras publicações que preenchessem os critérios de inclusão.

\subsection{Critérios de inclusão}

Foram considerados os seguintes critérios de inclusão: (i) artigos originais publicados em periódicos peer-reviewed com objetivo de verificar a relação entre o autoconceito e o rendimento acadêmico; (ii) estudos publicados entre janeiro de 2007 e junho de 2017; (iii) estudos quantitativos; (iv) amostras com estudantes de ensino médio, nível escolar equivalente em estudos internacionais ou idades equivalentes aos estudantes do ensino médio brasileiro. Somente estudos originais e transversais foram considerados na referida revisão. Contudo, optou-se por restringir a revisão aos estudos com amostra igual ou superior a 100 indivíduos. Tal critério tem sido adotado em revisões sistemáticas prévias (Chen, Beydoun, \& Wang, 2008; Vagetti et al., 2014) com o intuito de obter maior poder estatístico na detecção da associação entre variáveis e maior confiabilidade na interpretação dos achados.

Foram incluídos, então, estudos que contivessem procedimentos tais como: autorrelatos de autoconceito, questionários, inventários e escalas de autoconceito. $\mathrm{O}$ desfecho analisado em todos os estudos foi o rendimento acadêmico objetivo ou percebido: notas, conceitos ou construtos de competência percebida.

\subsection{Avaliação DA QUALIDAde DOS RESULTADOS}

Para avaliação da qualidade dos estudos, considerou-se a checklist Strengthening the Reporting of Observational Studies in Epidemiology (STROBE) (Von Elm et al., 2014). A lista de checagem do STROBE contém 22 itens, com recomendações sobre o que deve ser incluído em uma descrição mais precisa e completa de estudos observacionais. Todas as questões foram codificadas como 0 (zero) (representando qualidade inadequada) ou 1 (um) (representando qualidade adequada). Os escores de qualidade do estudo poderiam variar entre $0 \mathrm{e}$ 22 pontos. No caso de eventuais diferenças entre os dois revisores, na avaliação dos artigos foram realizadas reavaliações do artigo até ambos os revisores concordarem com a avaliação.

\subsection{ConsistênCIA E DIREÇÃo DA ASSOCIAÇÃo ENTRE AUTOCONCEITO E RENDIMENTO ACADÊMICO}

Com base nos estudos revisados, foi realizada uma avaliação da consistência da associação entre o autoconceito e o rendimento acadêmico. A força de evidências foi classificada em três categorias: consistente ( $\geq 60 \%$ dos estudos); moderada (30\%-59\% dos estudos); ou inconsistente ( $\leq 30 \%$ dos estudos). Essa classificação foi adotada em revisão prévia (Strong et al., 2005).

\subsection{EXTRAÇÃO DOS DADOS}

Para os estudos incluídos na presente revisão, os seguintes dados foram extraídos: país e local do estudo; tipo (estudantes do ensino médio brasileiro ou equivalente internacional) e tamanho da amostra; instrumentos de medida do autoconceito e rendimento acadêmico; variáveis de ajuste; e principais resultados. 


\section{RESULTADOS}

A Figura 1 apresenta o fluxograma descrevendo o processo de busca e seleção dos estudos. Foram identificados 1405 artigos potencialmente relevantes para esta revisão. Após a análise dos títulos, 68 estudos foram selecionados para leitura dos resumos. Foram considerados relevantes, de acordo com a leitura do resumo, 26 estudos, sendo selecionados para leitura do texto na íntegra. Destes, 18 estudos $(69,2 \%)$ foram excluídos por não atenderem aos critérios de elegibilidade: 12 estudos utilizaram faixa etária superior ou inferior ao da população-alvo e seis estudos não apresentaram o rendimento acadêmico objetivo ou percebido como desfecho da associação. Portanto, a busca eletrônica gerou oito estudos relevantes para essa revisão sistemática. Adicionalmente, dois estudos foram incluídos durante a leitura das referências dos artigos selecionados, completando, assim, 10 estudos analisados nesta revisão.

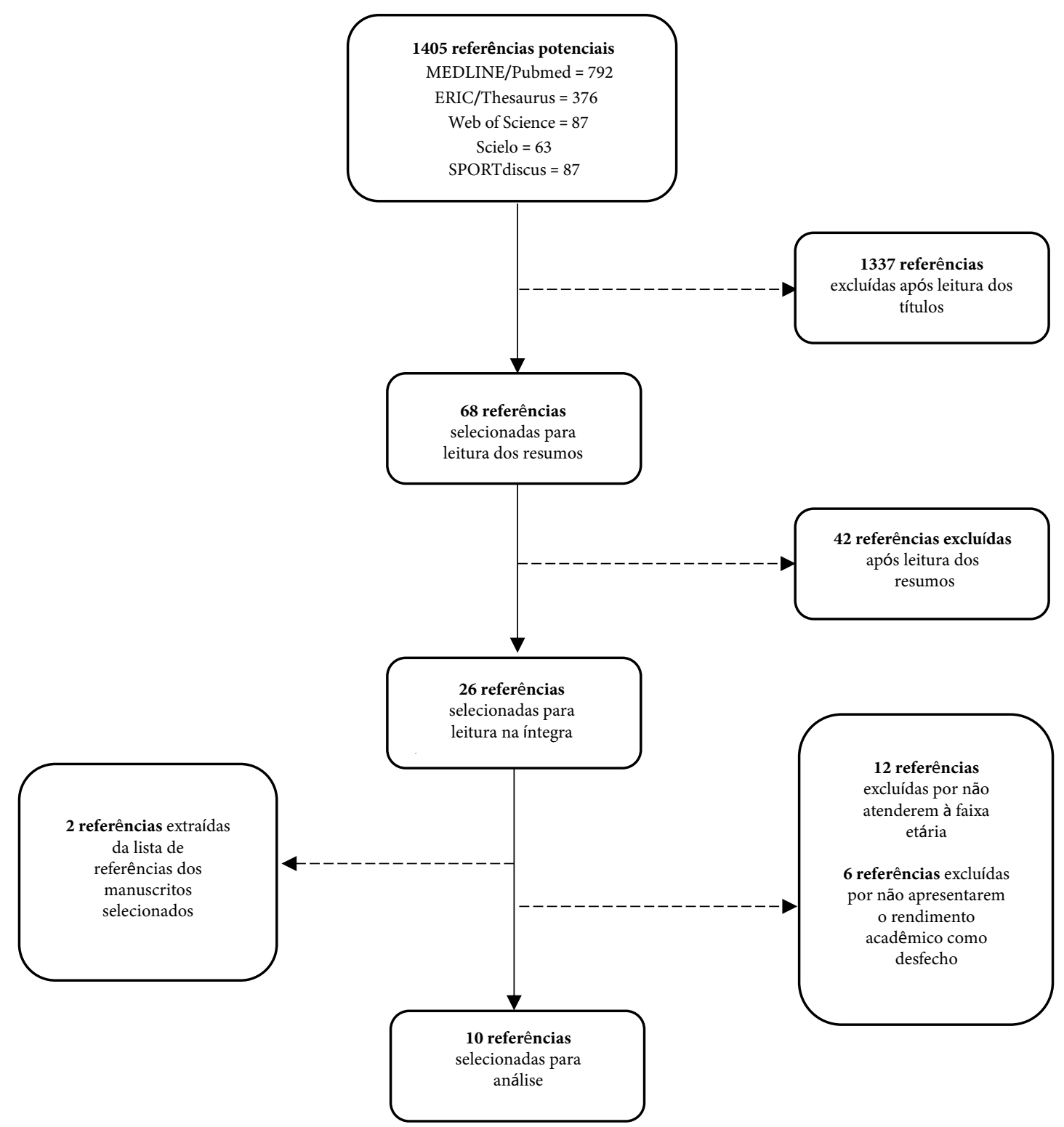

FI GU RA 1 - Fluxograma de busca e seleção dos estudos incluídos na revisão 
O Quadro 1 apresenta os estudos incluídos na revisão e seus principais achados quanto à relação entre o autoconceito e o desempenho acadêmico.

QUA DRO 1 - Estudos incluídos na revisão de literatura e seu(s) contributo(s) para a análise da relação entre autoconceito e desempenho acadêmico

\begin{tabular}{|c|c|c|c|c|c|c|}
\hline $\begin{array}{c}\text { Autor/ano; } \\
\text { Pontos na } \\
\text { avaliação de } \\
\text { qualidade } \\
\text { STROBE }\end{array}$ & $\begin{array}{c}\text { País / Data da } \\
\text { coleta }\end{array}$ & Amostra & $\begin{array}{l}\text { Instrumentos } \\
\text { de avaliação do } \\
\text { autoconceito }\end{array}$ & $\begin{array}{c}\text { Instrumentos } \\
\text { de avaliação } \\
\text { do rendimento } \\
\text { acadêmico }\end{array}$ & $\begin{array}{c}\text { Outras } \\
\text { variáveis } \\
\text { independentes }\end{array}$ & Principais achados \\
\hline $\begin{array}{l}\text { Awan, Noureen, } \\
\text { \& Naz, } 2011 \\
\text { STROBE ( } 20 \\
\text { pontos) }\end{array}$ & Paquistão / 2009 & $\mid \begin{array}{c}318 \text { estudantes } \\
\text { do ensino } \\
\text { médio, } 146 \\
\text { meninos e } 172 \\
\text { meninas }\end{array}$ & $\begin{array}{c}\text { Academic Self- } \\
\text { Description } \\
\text { Questionnaire II } \\
\text { (ASDQ II) }\end{array}$ & $\begin{array}{c}\text { General } \\
\text { Achievement } \\
\text { Goal Orientation } \\
\text { Scale }\end{array}$ & $\begin{array}{l}\text { Motivação } \\
\text { acadêmica }\end{array}$ & $\begin{array}{l}\text { O autoconceito e a motivação acadêmica } \\
\text { foram preditores do desempenho } \\
\text { acadêmico em Matemática }(\mathrm{R} 2=0,381) \text { e } \\
\text { em Língua Inglesa }(\mathrm{R} 2=0,242) ; \\
\mathrm{O} \text { autoconceito relacionado à } \\
\text { Matemática e também à Língua Inglesa foi } \\
\text { superior no sexo feminino. }\end{array}$ \\
\hline $\begin{array}{l}\text { Lone \& Lone, } \\
\quad 2016 \\
\begin{array}{l}\text { STROBE (18 } \\
\text { pontos) }\end{array}\end{array}$ & Índia / não cita & $\begin{array}{c}248 \text { estudantes } \\
\text { do último } \\
\text { ano do ensino } \\
\text { médio } 128 \\
\text { meninos e } 120 \\
\text { meninas }\end{array}$ & $\begin{array}{l}\text { Self-Concept tool } \\
\text { by R.K. Saraswat } \\
\text { (English version) }\end{array}$ & \begin{tabular}{|c|} 
Escore obtido na \\
base de dados \\
do desempenho \\
acadêmico da \\
escola, pelas notas \\
do estudante
\end{tabular} & Não houve & $\begin{array}{l}\text { Correlação positiva entre o autoconceito } \\
\text { e o desempenho acadêmico para a amostra } \\
\text { geral }(\mathrm{r}=0,152) \text {; } \\
\text { Inexistência de correlação entre o } \\
\text { autoconceito e o desempenho acadêmico } \\
\text { entre as meninas; } \\
\text { Correlação positiva entre o autoconceito } \\
\text { e o desempenho acadêmico entre os } \\
\text { meninos }(\mathrm{r}=0,187) \text {. }\end{array}$ \\
\hline $\begin{array}{l}\text { Areepattamannil } \\
\text { \& Freeman, } 2008 \\
\text { STROBE (20 } \\
\text { pontos) }\end{array}$ & $\begin{array}{c}\text { Canadá / não } \\
\text { cita }\end{array}$ & $\mid \begin{array}{c}248 \text { estudantes } \\
\text { do último } \\
\text { ano do ensino } \\
\text { médio } 128 \\
\text { meninos e } 120 \\
\text { meninas }\end{array}$ & $\begin{array}{c}\text { Academic Self- } \\
\text { Description } \\
\text { Questionnaire II } \\
\text { (ASDQ II) }\end{array}$ & $\begin{array}{c}\text { Escore } \\
\text { autorrelatado } \\
\text { da média de } \\
\text { desempenho } \\
\text { nas disciplinas } \\
\text { acadêmicas }\end{array}$ & $\begin{array}{l}\text { Motivação } \\
\text { acadêmica }\end{array}$ & $\begin{array}{l}\text { A média das notas em Língua Inglesa } \\
\text { apontou correlação com o autoconceito } \\
\text { verbal somente entre os nativos (r=0,51); } \\
\text { A média das notas em Matemática } \\
\text { apontou correlação com o autoconceito } \\
\text { matemático, entre nativos (r=0,66) e } \\
\text { imigrantes }(\mathrm{r}=0,70) \text {; } \\
\text { A média geral das notas apresentou } \\
\text { correlação com autoconceito acadêmico } \\
\text { entre os imigrantes; } \\
\text { A análise de regressão apontou } \\
\text { predição do autoconceito verbal entre } \\
\text { hativos ( } \beta=0,443) \text { e imigrantes ( } \beta=0,252) \\
\text { o autoconceito acadêmico entre nativos } \\
(\beta=0,194) \text { e imigrantes }(\beta=0,269) \text { para o } \\
\text { rendimento acadêmico em Língua Inglesa; } \\
\text { O autoconceito matemático foi preditor } \\
\text { do rendimento acadêmico em Matemática } \\
\text { nntre nativos }(\beta=0,629) \text { e imigrantes } \\
\quad(\beta=0,648) \text {; } \\
\text { A motivação extrínseca foi preditora } \\
\text { do rendimento acadêmico geral entre os } \\
\text { imigrantes }(\beta=0,197) \text {. }\end{array}$ \\
\hline $\begin{array}{l}\text { Stocker \& Faria, } \\
2012 \\
\text { STROBE (20 } \\
\text { pontos) }\end{array}$ & $\begin{array}{c}\text { Portugal / não } \\
\text { cita }\end{array}$ & $\begin{array}{l}1794 \\
\text { estudantes } \\
\text { de escolas } \\
\text { públicas e } \\
\text { privadas }\end{array}$ & $\begin{array}{c}\text { Academic Self- } \\
\text { Description } \\
\text { Questionnaire III } \\
\text { (ASDQ III) }\end{array}$ & $\begin{array}{c}\text { Autoeficácia } \\
\text { acadêmica medida } \\
\text { pela Escala de } \\
\text { Autoeficácia } \\
\text { Académica (EAEA) }\end{array}$ & Não houve & $\begin{array}{l}\text { A medida de autoeficácia em Língua } \\
\text { Portuguesa apresentou correlação com } \\
\text { o autoconceito verbal ( } \mathrm{r}=0,61) \text { e com o } \\
\text { autoconceito acadêmico }(\mathrm{r}=0,52) ; \\
\text { A medida de autoeficácia em } \\
\text { Matemática apresentou correlação com } \\
\text { o autoconceito verbal }(\mathrm{r}=0,22) \text {, com o } \\
\text { autoconceito acadêmico }(\mathrm{r}=0,47) \text { e com o } \\
\text { autoconceito matemático }(\mathrm{r}=0,76) \text {; } \\
\text { A medida de autoeficácia Escolar Geral } \\
\text { apresentou correlação com o autoconceito } \\
\text { verbal ( } \mathrm{r}=0,43) \text {, com o autoconceito } \\
\text { acadêmico ( } \mathrm{r}=0,34) \text { e com o autoconceito } \\
\text { matemático }(\mathrm{r}=0,67) \text {. }\end{array}$ \\
\hline
\end{tabular}

(continua na página seguinte) 


\begin{tabular}{|c|c|c|c|c|c|c|}
\hline $\begin{array}{l}\text { Castejón, Gilar, } \\
\text { Veas, \& Miñano, } \\
2016 \\
\text { STROBE (21 } \\
\text { pontos) }\end{array}$ & $\begin{array}{l}\text { Espanha / não } \\
\text { cita }\end{array}$ & $\begin{array}{c}1400 \\
\text { estudantes do } \\
\text { ensino médio }\end{array}$ & $\begin{array}{c}\text { Self-Concept } \\
\text { Evaluation Scale for } \\
\text { Adolescents (ESEA-2) }\end{array}$ & $\begin{array}{l}\text { Escore gerado por } \\
\text { meio das notas } \\
\text { escolares anuais }\end{array}$ & Não houve & $\begin{array}{l}\text { Estudantes com elevado desempenho } \\
\text { acadêmico tiveram valores superiores } \\
\text { de autoconceito matemático }(\mathrm{F}=29,55 ; \\
\mathrm{p}<0,001) \text {, verbal }(\mathrm{F}=70,33 ; \quad \mathrm{p}<0,001) \\
\text { acadêmico geral }(\mathrm{F}=126,95 ; \quad \mathrm{p}<0,001) \\
\mathrm{e} \text { autoconceito geral pessoal }(\mathrm{F}=32,71 ; \\
\mathrm{p}<0,001) \text {, relativamente a estudantes } \\
\text { com desempenho normal, e estes tiveram } \\
\text { valores superiores comparados aos alunos } \\
\text { com baixo desempenho escolar. }\end{array}$ \\
\hline $\begin{array}{l}\text { Abruzzo, Lenis, } \\
\text { Romero, Maser, \& } \\
\text { Morote, } 2016 \\
\text { STROBE (18 } \\
\text { pontos) }\end{array}$ & \begin{tabular}{|c} 
Estados Unidos \\
$/ 2007$
\end{tabular} & $\begin{array}{c}1284 \text { alunos de } \\
\text { duas escolas } \\
\text { do subúrbio de } \\
\text { Long Island }\end{array}$ & $\begin{array}{c}\text { Survey instrument } \\
\text { developed by Maser } \\
\text { (2007) }\end{array}$ & $\begin{array}{c}\text { Escore gerado por } \\
\text { meio das notas } \\
\text { escolares anuais }\end{array}$ & \begin{tabular}{|c|} 
Participação \\
em atividades \\
extracurriculares
\end{tabular} & $\begin{array}{c}\mathrm{O} \text { autoconceito acadêmico e a } \\
\text { participação esportiva extracurricular, em } \\
\text { conjunto, explicaram } 33 \%(\mathrm{R} 2=0,33) \text { da } \\
\text { variância do rendimento acadêmico em } \\
\text { Língua Inglesa; } \\
\text { O autoconceito acadêmico e a } \\
\text { participação esportiva extracurricular, em } \\
\text { conjunto, explicaram } 23 \%(\mathrm{R} 2=0,23) \text { da } \\
\text { variância do rendimento acadêmico em } \\
\text { Matemática; } \\
\text { O autoconceito acadêmico e a } \\
\text { participação esportiva extracurricular, em } \\
\text { conjunto, explicaram } 32 \%(\mathrm{R} 2=0,32) \text { da } \\
\text { variância do rendimento acadêmico médio } \\
\text { geral. }\end{array}$ \\
\hline $\begin{array}{l}\text { Scherer \& } \\
\text { Scherer, } 2013 \\
\text { STROBE (21 } \\
\text { pontos) }\end{array}$ & $\begin{array}{c}\text { Alemanha / não } \\
\text { cita }\end{array}$ & $\begin{array}{l}459 \text { estudantes } \\
\text { do ensino } \\
\text { médio técnico } \\
\text { (Química) de } \\
\text { cinco escolas } \\
\text { federais }\end{array}$ & $\begin{array}{c}\text { Quatro itens do } \\
\text { German version of } \\
\text { the PISA student } \\
\text { questionnaire, que } \\
\text { versam sobre o } \\
\text { autoconceito geral } \\
\text { e específico em } \\
\text { Química }\end{array}$ & $\begin{array}{c}\text { Escore gerado por } \\
\text { meio das notas } \\
\text { escolares anuais, em } \\
\text { Química }\end{array}$ & \begin{tabular}{|c|} 
Envolvimento \\
com ciências; \\
atividades práticas \\
em Química
\end{tabular} & $\begin{array}{l}\text { O autoconceito acadêmico geral teve } \\
37 \% \text { de explicação da sua variância } \\
\text { (R2 }=0,37) \text { pelo modelo que incluiu o } \\
\text { desempenho acadêmico geral em ciências, } \\
\text { o desempenho em Biologia, Química, } \\
\text { Língua Alemã e Matemática; } \\
\text { O autoconceito específico em Química } \\
\text { teve } 40 \% \text { de explicação de sua variância } \\
\text { (R2 }=0,40) \text { pelo modelo que incluiu o } \\
\text { envolvimento em ciências, atividades } \\
\text { práticas em Química e desempenho em } \\
\text { Química }\end{array}$ \\
\hline $\begin{array}{c}\text { Jansen, Scherer, \& } \\
\text { Schroeders, } 2015 \\
\text { STROBE }(21 \\
\text { pontos) }\end{array}$ & $\begin{array}{l}\text { Alemanha / } \\
2006\end{array}$ & $\begin{array}{c}4891 \\
\text { estudantes de } \\
\text { ensino médio } \\
\text { de } 226 \text { escolas }\end{array}$ & $\begin{array}{c}\text { German version of } \\
\text { the PISA student } \\
\text { questionnaire }\end{array}$ & $\mid \begin{array}{c}\text { Resultado do PISA } \\
\text { em ciências }\end{array}$ & $\begin{array}{l}\text { Autoeficácia } \\
\text { acadêmica }\end{array}$ & $\begin{array}{l}\text { No modelo } 1 \text { de regressão, o autoconceito } \\
\text { específico em ciências explicou } 8 \% \text { da } \\
\text { variância }(\mathrm{R} 2=0,08) \text { do desempenho em } \\
\text { ciências; } \\
\text { No modelo 2, a autoeficácia acadêmica } \\
\text { em ciências explicou } 28 \% \text { da variância } \\
\text { (R2=0,28) do desempenho em ciências; } \\
\text { No modelo } 3 \text { o autoconceito e a } \\
\text { autoeficácia explicaram } 28 \% \text { da variância } \\
\text { (R2=0,28) do desempenho em ciências, } \\
\text { ou seja, o autoconceito não explicou } \\
\text { incrementos de valores na variância. }\end{array}$ \\
\hline $\begin{array}{l}\text { Álvarez et al., } \\
\quad 2015 \\
\text { STROBE (19 } \\
\text { pontos) }\end{array}$ & $\begin{array}{l}\text { Espanha / não } \\
\text { cita }\end{array}$ & $\begin{array}{c}502 \text { estudantes } \\
\text { do ensino } \\
\text { secundário } \\
\text { obrigatório } \\
\text { espanhol }\end{array}$ & $\begin{array}{c}\text { Self-Concept } \\
\text { Evaluation Scale for } \\
\text { Adolescents (ESEA-2) }\end{array}$ & $\begin{array}{l}\text { Escore gerado } \\
\text { por meio da } \\
\text { média das notas } \\
\text { nas disciplinas } \\
\text { obrigatórias }\end{array}$ & $\begin{array}{c}\text { Envolvimento } \\
\text { familiar percebido }\end{array}$ & $\begin{array}{l}\text { As variáveis de envolvimento familiar } \\
\text { mostraram-se diretamente pouco } \\
\text { associadas com o rendimento acadêmico; } \\
\text { As variáveis de envolvimento familiar } \\
\text { mostraram-se diretamente associadas com } \\
\text { o autoconceito; } \\
\text { O autoconceito respondeu em } 49 \% \\
(\mathrm{R} 2=0,49) \text { à variância do rendimento } \\
\text { acadêmico. }\end{array}$ \\
\hline $\begin{array}{l}\text { Veas, Castejón, } \\
\text { Gilar, \& Miñano, } \\
2015 \\
\text { STROBE (21 } \\
\text { pontos) }\end{array}$ & $\begin{array}{c}\text { Espanha / 2011- } \\
2012\end{array}$ & \begin{tabular}{|c}
1456 \\
estudantes \\
do segundo \\
ano do ensino \\
secundário \\
obrigatório \\
espanhol
\end{tabular} & $\begin{array}{c}\text { Self-Concept } \\
\text { Evaluation Scale for } \\
\text { Adolescents (ESEA-2) }\end{array}$ & $\begin{array}{l}\text { Escore gerado } \\
\text { por meio da } \\
\text { média das notas } \\
\text { nas disciplinas } \\
\text { obrigatórias }\end{array}$ & \begin{tabular}{|c|} 
Habilidade \\
intelectual, \\
orientação \\
de metas e \\
envolvimento \\
familiar percebido
\end{tabular} & $\begin{array}{l}\text { O modelo incluindo todas as variáveis } \\
\text { independentes respondeu por } 56 \% \text { da } \\
\text { variância do rendimento acadêmico; } \\
\text { A maior parte do resultado foi explicado } \\
\text { pelo autoconceito, } 47 \%(\mathrm{R} 2=0,47) \text {. }\end{array}$ \\
\hline
\end{tabular}




\subsection{QUALIDADE DOS ESTUDOS}

$\mathrm{Na}$ avaliação, segundo a lista de checagem do STROBE (Von Elm et al., 2014), verificou-se que nenhum estudo atingiu a pontuação máxima de 22 pontos; enquanto dois estudos obtiveram uma menor pontuação (18 pontos), outros quatro chegaram à pontuação de 21 , como pode observar-se no Quadro 1.

\subsection{CARACTERÍSTICAS GERAIS DOS EST U D O S}

Em relação aos 10 estudos incluídos na revisão, dois foram realizados na Alemanha (Jansen, Scherer, \& Schroeders, 2015; Scherer \& Scherer, 2013). Sendo que um dos integrantes - Scherer - compõe a autoria dos dois trabalhos, nesses estudos há utilização de variáveis e análises similares; outros três foram desenvolvidos na Espanha (Álvarez et al., 2015; Castejón, Gilar, Veas, \& Miñano, 2016; Veas, Castejón, Gilar, \& Miñano, 2015). Os outros estudos foram publicados por grupos de Portugal (Stocker \& Faria, 2012), asiáticos (Awan, Noureen, \& Naz, 2011; Lone \& Lone, 2016) e da América do Norte (Abruzzo, Lenis, Romero, Maser, \& Morote, 2016; Areepattamannil \& Freeman, 2008). Somente um dos estudos foi publicado anteriormente a 2010 (Areepattamannil \& Freeman, 2008). Todos eles incluíram estudantes de ambos os sexos na amostra geral.

Do total de trabalhos, apenas um não realizou análises de relação entre o autoconceito e o rendimento acadêmico, seja pelo teste de correlação ou por algum tipo de regressão (Castejón et al., 2016). Entre os estudos que fizeram análise preditiva do rendimento acadêmico, sete utilizaram outras variáveis explicativas em conjunto com o autoconceito (Abruzzo et al., 2016; Álvarez et al., 2015; Areepattamannil \& Freeman, 2008; Awan et al., 2011; Jansen et al., 2015; Scherer \& Scherer, 2013; Veas et al., 2015). Destes, além do autoconceito, dois trabalhos utilizaram a motivação acadêmica como variável explicativa (Areepattamannil \& Freeman, 2008; Awan et al., 2011); dois, o envolvimento familiar percebido (Álvarez et al., 2015; Veas et al., 2015); um trabalho utilizou a participação em atividades extracurriculares (Abruzzo et al., 2016); outro, o envolvimento com ciências e atividades práticas da disciplina (Scherer \& Scherer, 2013); um utilizou orientações de metas e habilidades intelectuais (Veas et al., 2015); e outro utilizou a autoeficácia acadêmica (Jansen et al., 2015).

Oito artigos apresentaram, como variável independente, outros tipos de autoconceito, além do geral. Quatro estudos apresentaram os autoconceitos acadêmico, verbal e matemático (Areepattamannil \& Freeman, 2008; Castejón et al., 2016; Stocker \& Faria, 2012; Veas et al., 2015); dois estudos utilizaram medidas dos autoconceitos específicos em Química e Ciências (Jansen et al., 2015; Scherer \& Scherer, 2013); um trabalho utilizou o autoconceito em Língua Inglesa e Matemática (Awan et al., 2011); e um dos estudos utilizou somente o autoconceito acadêmico (Álvarez et al., 2015).

\subsection{MEDIDAS DE AUTOCONCEITO}

O Academic Self-Description Questionnaire II (ASDQ II) e o Self-Concept Evaluation Scale for Adolescents (ESEA-2) foram os instrumentos aplicados em mais do que um trabalho (Álvarez et al., 2015; Areepattamannil \& Freeman, 2008; Awan et al., 2011; Castejón et al., 2016; Veas et al., 2015). Os outros estudos aplicaram questionários distintos para obtenção dessa variável: Lone e Lone (2016) utilizaram o Self-Concept tool by R. K. Saraswat (English version), Stocker e Faria (2012) o Academic Self-Description Questionnaire III (ASDQ III), Abruzzo et al. (2016) o Survey instrument developed by Maser (2007); Scherer \& Scherer (2013) utilizaram questões específicas da versão 
alemã do Programme for International Student Assessement (PISA), assim como Jansen et al. (2015).

Para obtenção das medidas de rendimento acadêmico dos estudantes, foram utilizadas quatro metodologias diferentes. Mais de metade dos trabalhos (seis) utilizaram medidas das médias anuais das notas obtidas pelos estudantes em determinadas disciplinas (Abruzzo et al., 2016; Álvarez et al., 2015; Castejón et al., 2016; Lone \& Lone, 2016; Scherer \& Scherer, 2013; Veas et al., 2015). Outro trabalho obteve as notas dos estudantes por meio do autorrelato destes (Areepattamannil \& Freeman, 2008). Os outros três estudos adquiriram essa medida por meio da aplicação de algum teste ou escala. Awan et al. (2011) aplicaram a General Achievement Goal Orientation Scale. Já Stocker e Faria (2012) utilizaram a Escala de Autoeficácia Académica (EAEA). Por fim, Jansen et al. (2015) utilizaram o resultado da versão alemã do PISA.

\subsection{SUMÁRIO DE EVIDÊNCIAS DA ASSOCIAÇÃO ENTRE AUTOCONCEITO E RENDIMENTO ACADÊMICO}

Somente um dos estudos não evidenciou relação do autoconceito com o rendimento acadêmico (Jansen et al., 2015). Os outros nove trabalhos apresentaram relação positiva dos mesmos. Esse valor corresponde a $90 \%$ dos artigos, que apresentaram tal relação. Segundo a metodologia adotada para evidenciar consistência da associação ( $\geq 60 \%$ dos estudos), essa relação foi classificada como consistente (Strong et al., 2005).

\section{Discuss Ão}

\subsection{CARATERÍSTICAS DOS ESTUDOS}

Apesar de o estudo do autoconceito em ambiente escolar ser um tema bastante debatido na literatura, com trabalhos publicados há décadas e outros que continuam sendo realizados, essa revisão apresentou somente 10 estudos com as características selecionadas para inclusão (Abruzzo et al., 2016; Álvarez et al., 2015; Areepattamannil \& Freeman, 2008; Awan et al., 2011; Castejón et al., 2016; Jansen et al., 2015; Lone \& Lone, 2016; Scherer \& Scherer, 2013; Stocker \& Faria, 2012; Veas et al., 2015). Entre os estudos que debatem essa relação, parte relevante das publicações são realizadas com amostras com crianças e somente um número reduzido de referências analisou o autoconceito entre adolescentes. Esse quantitativo de somente 10 trabalhos surpreende, uma vez que o autoconceito é tratado como uma das principais variáveis afetivo-emocionais relacionadas ao sucesso em diversas dimensões da vida do indivíduo e a adolescência é vista como um período sensível para alterações de percepção que podem afetar o autoconceito do indivíduo (Cia \& Barham, 2008).

Observou-se que a maioria das pesquisas incluídas nessa revisão foi realizada em países desenvolvidos, sendo somente dois os estudos realizados fora do eixo Europa/América do Norte (Awan et al., 2011; Lone \& Lone, 2016). Cabe citar que, baseados nos trabalhos iniciais de Shavelson, Hubner, e Stanton (1976), pesquisadores portugueses e espanhóis se empenharam no estudo do autoconceito em diferentes contextos, desenvolvendo e adaptando, inclusive, diversos instrumentos de medida dessa variável, para diferentes populações (García-Grau, Pérez, Moreno, \& Prado-Gascó, 2014; Pipa \& Peixoto, 2011; Veiga, 2005). Fica evidente a expertise desses grupos sobre a discussão do autoconceito ao se observar que, nessa revisão, além dos quatro estudos publicados por portugueses e espanhóis, outros dois trabalhos utilizaram instrumentos desenvolvidos por pesquisadores desses países (Areepattamannil \& Freeman, 2008; Awan et al., 2011).

Skaalvik e Bong (2003) citam que o 
autoconceito acadêmico pode ser definido como o autoconceito acadêmico geral ou específico para cada área técnica. Com exceção do estudo de Scherer e Scherer (2013), que utilizou amostra de estudantes de cursos técnicos e avaliou também o autoconceito direcionado à área técnica específica, todos os outros estudos abordaram as dimensões verbal, matemática e geral do autoconceito. Nesse sentido, opta-se pela avaliação do autoconceito verbal e matemático, além do geral, como forma de contemplar os domínios interpretativos e reflexivos, bem como de raciocínio lógico matemático.

Somente um dos estudos não contemplou o rendimento acadêmico de forma quantitativa: notas anuais, escala de conhecimento ou resultado em testes governamentais (Stocker \& Faria, 2012). Apesar das discussões pedagógicas acerca da função e eficácia das notas, rankings ou escalas como forma de avaliação da aprendizagem, não se observam outras metodologias para obtenção desses parâmetros em grandes amostras, como as identificadas nos estudos dessa revisão. Entretanto, é importante salientar que essas medidas se restringem ao resultado do produto final, a medida, e não ao processo de aprendizagem pelo qual o estudante passou, igualmente importante.

\subsection{RELAÇÃo DO AUTOCONCEITO COM O} RENDIMENTO ACADÊMICO

Entre os 10 estudos que incluíram essa revisão, somente um não identificou relação do autoconceito com o rendimento acadêmico (Jansen et al., 2015). No estudo citado, a autoeficácia acadêmica em Matemática explicou o rendimento acadêmico de forma mais efetiva do que o autoconceito, contrariamente à hipótese formulada pelos próprios autores. Segundo esses pesquisadores, a explicação para o ocorrido pode estar no fato de os itens formulados para avaliar a autoeficácia serem tipicamente abordados em testes matemáticos. Esses itens estão contemplados também na versão alemã do Programme for International Student Assessment (PISA, 2006), da qual foram extraídos os resultados de rendimento acadêmico em matemática. Dessa forma, Stankov et al. (2012) especularam que a similaridade entre os itens de medida da autoeficácia acadêmica e os itens do teste de conhecimento pode ter moderado a relação entre as variáveis. Para os autores, se o rendimento fosse medido de forma diferente relativamente ao PISA, uma relação menor poderia ser esperada.

Todos os outros estudos identificaram relação do autoconceito com o rendimento acadêmico. Awan et al. (2011) concluíram que um autoconceito positivo pode promover aumento no nível de motivação dos estudantes, que, consequentemente, impacta na melhora de seu desempenho acadêmico. Nessa perspectiva, a atitude de pais, colegas e professores pode ser determinante para melhora ou piora do autoconceito do estudante, mediado pela motivação. Diante disto, os autores concluem e salientam a importância do professor no desenvolvimento de estratégias que possam auxiliar no aspecto motivacional com foco na performance dos estudantes.

Lone e Lone (2016) sugerem, a partir da correlação identificada entre o autoconceito geral e o rendimento acadêmico, que se deve levar em consideração o planejamento escolar realizado pela instituição, em que se dê oportunidade de atividades guiadas e lúdicas dentro e fora da escola, que vise o aumento do autoconceito. Ainda na escola, os professores poderiam desenvolver atividades de classe, de forma que oportunizassem a participação dos estudantes de igual forma. Já a família apresenta o papel de promover o suporte e um ambiente propício à liberdade de expressão de seus pensamentos e sentimentos. Nessa perspectiva, a experiência de destemor se reflete em sua personalidade em 
relação a um alto autoconceito.

Considerando as características sociodemográficas do Canadá e o grande número de imigrantes que se instalam no país, há preocupação de proporcionar bem-estar a esses indivíduos. $\mathrm{O}$ desenvolvimento desse bem-estar passa pela preocupação com o autoconceito, principalmente de crianças e adolescentes que se inserem em uma nova cultura. Foi nesse contexto que Areepattamannil e Freeman (2008) realizaram um estudo em que se concluiu que o autoconceito verbal e matemático explicou o rendimento acadêmico de estudantes nativos e imigrantes de forma similar. Para os autores, esse resultado pode ser explicado pela política seletiva de imigração, o que resulta em uma população de imigrantes com melhor educação. Além disso, no mesmo trabalho, se observam valores superiores na avaliação motivacional dos estudantes imigrantes, comparados aos nativos, e já foi apontada na literatura a relação positiva entre motivação e autoconceito (Awan et al., 2011).

No trabalho de construção de um novo instrumento, Stocker e Faria (2012) foram os únicos autores que utilizaram outro construto para avaliar o desempenho do estudante, que não as notas escolares ou testes, nesta revisão. Esses autores verificaram relações positivas entre o autoconceito e a competência percebida pelos estudantes. Outros estudos apontaram que a competência acadêmica percebida pode refletir, significativamente, o desempenho acadêmico dos estudantes, mostrando-se, então, uma alternativa interessante para investigação dessa associação, além das notas e escores (Leme \& Marturano, 2014; Pasin, Paiva, \& Lannes, 2012). Na construção desse instrumento, Stocker e Faria (2012) discutiram por que foram abordados somente o autoconceito verbal e matemático, além do geral. Os autores justificam que, como em estudos prévios, existe uma relação importante entre os dois primeiros e o autoconceito geral acadêmico, o que eximiria a necessidade de obtenção dessa variável para cada área do conhecimento.

No estudo de Castejón et al. (2016) foi evidenciado que há maiores valores de autoconceito entre os estudantes com alto rendimento acadêmico, em comparação aos de médio e baixo desempenho; os autores sugerem que o sucesso dos estudantes com alto desempenho acadêmico está na quantidade e diversidade de estratégias de aprendizado que utilizam. Citam, ainda, que é necessária mais investigação para determinar se o menor autoconceito entre os estudantes com baixo rendimento acadêmico é resultado de uma compensação devido ao baixo autoconceito acadêmico e pessoal. $\mathrm{O}$ autoconceito dos estudantes com baixo rendimento apresentou perfil inferior aos de normal e alto rendimento em todas as dimensões pessoais e acadêmicas, inclusive no que diz respeito à relação com os pais, o que demanda atenção especial, uma vez que o autoconceito também é determinado pelas relações familiares do indivíduo (Lone \& Lone, 2016).

No estudo de Scherer e Scherer (2013) o autoconceito foi tratado como variável dependente e verificou-se que foi explicado pelo rendimento de disciplinas específicas. Este trabalho foi desenvolvido com uma amostra de estudantes alemães de escolas técnicas, no curso de Química. Para os autores, esse achado reforça a ideia de direcionamento de estudos do autoconceito específico para determinadas áreas, pois, nessa condição, o estudante tem uma percepção positiva do seu domínio e habilidade sobre o conteúdo, o que eleva seu nível de competência e, consequentemente, seu rendimento acadêmico.

Tanto Álvarez et al. (2015) quanto Veas et al. (2015), na Espanha, observaram em seus estudos que, apesar da relação entre o envolvimento familiar e o autoconceito dos estudantes, 
o envolvimento familiar pouco explicou a associação com o rendimento acadêmico dos estudantes. Entretanto, o autoconceito respondeu por $49 \%$ da variância do desfecho na pesquisa de Álvarez et al. (2015) e 47\% na de Veas et al. (2015). Em ambas as pesquisas, os autores argumentam que a família exerce grande influência sobre a conduta de aprendizagem do sujeito e, consequentemente, seu rendimento. As atitudes positivas, crenças e expectativas dos pais, acerca da capacidade e sucesso dos filhos, favorecem o desenvolvimento de um autoconceito acadêmico positivo e um tipo de motivação (intrínseca e extrínseca) para as tarefas escolares, que determinam, em parte, os resultados acadêmicos. Entretanto, nos dois estudos, o envolvimento familiar não se relacionou diretamente com o rendimento acadêmico, como aconteceu com o autoconceito.

3.3 OUTRAS VARIÁVEIS INDEPENDENTES EXPLICATIVAS

Apesar de os estudos tratarem o autoconceito como um dos principais construtos afetivoemocionais capazes de interferir no rendimento acadêmico do estudante, a maioria dos trabalhos incluídos na revisão utilizou outras variáveis em conjunto ao autoconceito, com o objetivo de explicar, de forma mais efetiva, o desfecho (Abruzzo et al., 2016; Álvarez et al., 2015; Areepattamannil \& Freeman, 2008; Awan et al., 2011; Jansen et al., 2015; Scherer \& Scherer, 2013; Veas et al., 2015).

Dois dos estudos inseridos na revisão utilizaram também a motivação acadêmica como variável independente da relação (Areepattamannil \& Freeman, 2008; Awan et al., 2011). Para esses pesquisadores, a motivação é altamente destacada como uma necessidade psicológica para desenvolver a autonomia do indivíduo. A autonomia, por sua vez, implica escolha de experiências, manutenção e regulação de seus comportamentos. Entretanto, no estudo de Areepattamannil e Freeman (2008), a motivação não foi preditiva do desempenho acadêmico, tanto em adolescentes nativos, quanto em imigrantes canadenses, diferentemente do que aconteceu no estudo de Awan et al. (2011), que verificaram que, em conjunto com o autoconceito, a motivação acadêmica explicou quase $40 \%$ da variação do rendimento acadêmico de estudantes paquistaneses. Para esses autores, a motivação acadêmica pode implicar a organização e empenho nos estudos.

Abruzzo et al. (2016) demonstraram a importância da participação dos estudantes em atividades extracurriculares oferecidas pela escola. Neste trabalho, a prática esportiva frequente e a participação em outras atividades organizadas em contraturno, como música, teatro, entre outras, aliadas ao autoconceito, explicaram mais de um terço da variância dos resultados acadêmicos de estudantes norteamericanos. Esses autores destacam o esporte como ambiente favorecedor do autoconceito, $\mathrm{o}$ que, por consequência, melhora o desempenho escolar.

Ainda na perspectiva das atividades práticas, Scherer e Scherer (2013) destacaram a importância do envolvimento em atividades relacionadas à ciência, na escola, bem como a participação em atividades práticas de Química, desenvolvidas pelo professor. Essas atividades, somadas ao autoconceito, foram preditivas do rendimento escolar de estudantes de cursos técnicos de Química alemães.

Jansen et al. (2015) destacam a autoeficácia acadêmica como uma variável mais importante do que o autoconceito, nessa relação, uma vez que, em seu estudo, somente a autoeficácia se mostrou associada com o desempenho acadêmico. Os autores sugerem que a autoeficácia é a percepção da habilidade do próprio estudante em realizar uma tarefa escolar específica, enquanto $\mathrm{o}$ autoconceito tem dimensões mais amplas; assim, 
a autoeficácia poderia afetar de forma mais direta o rendimento acadêmico.

Por fim, Álvarez et al. (2015) e Veas et al. (2015) apresentaram o envolvimento familiar como uma variável que também poderia explicar o rendimento acadêmico. Contudo, em ambos os estudos, essa variável teve pouca relação com o desempenho acadêmico. Ainda assim, no trabalho de Álvarez et al. (2015), foi possível perceber que o envolvimento familiar se relacionou, positivamente, com o autoconceito, que, por sua vez, explicou o rendimento acadêmico; ou seja, o envolvimento familiar pode responder de forma indireta, sendo mediado pelo autoconceito, na relação.

\subsection{LIMITAÇÕES DO ESTUDO}

Este estudo apresentou algumas limitações. Primeiramente, a limitação do tempo especificado parabusca:2007 a 2017. Alguns estudos relevantes podem ter sido publicados anteriormente a esse período, uma vez que o autoconceito é uma variável estudada há bastante tempo. Entretanto, ao delinear a pesquisa, definiu-se esse recorte para que retornassem trabalhos com sistemas de medida das variáveis mais próximos do que ocorre hoje. A despeito disso, por exemplo, anteriormente à década de 1980 era comum a utilização da escala de autoconceito de Piers \& Haris, desenvolvida por esses autores em 1964, com possibilidade de resposta dicotômica em "sim" ou "não" para ideias sobre si mesmo (Marx \& Winne, 1978). Percebeu-se, entretanto, com o avançar das pesquisas na área, a necessidade de adaptação dessa escala, que hoje tem pelo menos mais duas versões adaptadas e validadas, a PHCSCS-2 (Piers \& Hertzberg, 2002) e a versão portuguesa PHCSCS V1-6 (Veiga \& Domingues, 2012).

A busca de estudos também foi limitada à literatura peer-reviewed, não sendo incluídos dados não publicados, teses, dissertações e posicionamentos de instituições.

Possivelmente, a inclusão de alguma outra base de dados científicos no processo de busca retornaria um artigo diferente dos incluídos na revisão. Entretanto, as bases escolhidas são de ampla divulgação e concentram grande parte das referências sobre o tema.

\section{CONSIDERAÇÕES FINAIS}

Essa revisão sistemática da literatura sobre a relação entre o autoconceito e o rendimento acadêmico incluiu 10 estudos que entraram nos critérios determinados. A relação entre essas variáveis ficou evidente na grande maioria dos estudos. Somente um não identificou tal associação.

Outras variáveis se mostraram importantes na explicação do desfecho do rendimento acadêmico. Entre as variáveis analisadas pelos trabalhos incluídos na revisão, estiveram a motivação acadêmica, a prática de atividades extracurriculares, a autoeficácia acadêmica e o envolvimento dos pais. Apesar de importantes fatores, a influência do grupo de pares, a expectativa dos docentes, o ambiente escolar, entre outros, não foram abordados nesses estudos. Isso demonstra um campo para futuras pesquisas.

A partir do evidenciado, percebe-se a necessidade de mais estudos que contemplem essa relação com estudantes adolescentes, frente ao baixo número de trabalhos com essa população. Percebe-se também a necessidade de desenvolvimento de pesquisas que contemplem outros espaços escolares, visto que as atividades práticas e extracurriculares apresentaram bons resultados quanto ao autoconceito. Esses resultados podem auxiliar no trato pedagógico dos agentes educacionais, como forma de auxiliar em estratégias que favoreçam o desenvolvimento do autoconceito e, consequentemente, o rendimento escolar. 


\section{REFERÊ NCIAS}

Abruzzo, K. J., Lenis, C., Romero, Y. V., Maser, K. J., \& Morote, E.-S. (2016). Does participation in extracurricular activities impact student achievement? Journal for Leadership and Instruction, 15(1), 21-26.

Allen, J., Gregory, A., Mikami, A., Lun, J., \& Hamre, B. (2013). Observations of effective teacher-student interactions in secondary school classrooms: Predicting student achievement with the classroom assessment scoring system-secondary - ProQuest. School Psychology Review, 42(1), 76-98.

Álvarez, A., Suárez, N., Tuero, E., Núñez, J. C., Valle, A., \& Regueiro, B. (2015). Implicación familiar, autoconcepto del adolescente y rendimiento académico. European Journal of Investigation in Health, Psychology and Education, 5(3), 293-311.

Areepattamannil, S., \& Freeman, J. G. (2008). Academic achievement, academic selfconcept, and academic motivation of immigrant adolescents in the greater Toronto area secondary schools. Journal of Advanced Academics, 19(4), 700-743.

Awan, R., Noureen, G., \& Naz, A. (2011). A study of relationship between achievement motivation, self-concept and achievement in English and Mathematics at secondary level. International Education Studies, 4(3), 72-79.

Castejón, J. L., Gilar, R., Veas, A., \& Miñano, P. (2016). Differences in learning strategies, goal orientations, and self-concept between overachieving, normal-achieving, and underachieving secondary students. Frontiers in Psychology, 7, 14-38.

Chen, X., Beydoun, M. A., \& Wang, Y. (2008). Is sleep duration associated with childhood obesity? A systematic review and metaanalysis. Obesity, 16(2), 265-274.

Cia, F., \& Barham, E. J. (2008). Estabelecendo relação entre autoconceito e desempenho acadêmico de crianças escolares. Psico, 39(1), 21-27.

Covington, M. V.(2000). Goal theory, motivation, and school achievement: An integrative review. Annual Review of Psychology, 51(1), 171-200.

García-Grau, P., Pérez, D. A., Moreno, F. C., \& Prado-Gascó, V. J. (2014). Self-concept in preadolescence: A brief version of AF5 scale. Motriz: Revista de Educação Física, 20(2), 151157.

Hattie, J. (2014). Self-concept. New York and London: Psychology Press.

Hines, E. M., \& Holcomb-McCoy, C. (2013). Parental characteristics, ecological factors, and the academic achievement of African American males. Journal of Counseling \& Development, 91(1), 68-77.

Jansen, M., Scherer, R., \& Schroeders, U. (2015). Students' self-concept and self-efficacy in the sciences: Differential relations to antecedents and educational outcomes. Contemporary Educational Psychology, 41, 13-24.

Klapp, A. (2017). Does academic and social selfconcept and motivation explain the effect of grading on students' achievement? European Journal of Psychology of Education, 33(2), 355376.

Leme, V. B. R., \& Marturano, E. M. (2014). Preditores de comportamentos e competência acadêmica de crianças de famílias nucleares, monoparentais e recasadas. Psicologia: Reflexão e Crítica, 27(1), 153-162.

Lone, P. A., \& Lone, T. A. (2016). A study on relation between self-concept and academic achievement among secondary school students of Jammu District. Journal of Education and Practice, 7(31), 19-23.

Marx, R. W., \& Winne, P. H. (1978). Construct interpretations of three self-concept inventories. American Educational Research Journal, 15(1), 99-109.

Miranda, L., \& Almeida, L. S. (2009). As metas académicas como operacionalização da motivação do aluno. Educação Temática Digital, 10, 36-61.

Oxford Centre for Evidence-Based Medicine (2016). Levels of evidence working group. The Oxford levels of evidence 2. Disponível em: 
https://www.cebm.net/index.aspx?o=5653 (acesso em: 05/03/2018).

Pasin, E. B., Paiva, M. G. V., \& Lannes, D. R. C. (2012). Associação entre fatores psicológicos e relacionais e o rendimento escolar no ensino fundamental. Avaliação Psicológica, 11(2), 275-286.

Piers, E. V., \& Harris, D. (1964). Age and others correlates of self-concept in children. Journal of Educational Psychology, 55(2), 91-95.

Piers, E. V., \& Herzberg, D. (2002). Piers-Harris Children's Self-Concept Scale (2a ed.). Wilshire Boulevard, CA: Western Psychological Services.

Pipa, J., \& Peixoto, F. J. B. (2011). Adaptação da escala de autoconceito artístico para estudantes do ensino secundário. In A. S. Ferreira, A. Verhaeghe, D. R. Silva, L. S. Almeida, R. Lima, \& S. Fraga (Eds.), Actas do VIII Congresso Iberoamericano de Avaliação/Evaluación Psicológica. XV Conferência Internacional Avaliação Psicológica: Formas e Contextos (pp. 668-678). Lisboa: Sociedade Portuguesa de Psicologia.

Purkey, W. W. (1970). Self-concept and school achievement. Oxford, England: Prentice-Hall.

Ribeiro, J. L. P. (2014). Revisão de investigação e evidência científica. Psicologia, Saúde \& Doenças, 15(3), 671-682.

Scherer, R., \& Scherer, R. (2013). Further evidence on the structural relationship between academic self-concept and self-efficacy: On the effects of domain specificity. Learning and Individual Differences, Complete 28, 9-19.

Shavelson, R. J., Hubner, J. J., \& Stanton, G. C. (1976). Self-concept: Validation of construct interpretations. Review of Educational Research, 46(3), 407-441.

Skaalvik, E., \& Bong, M. (2003). Self-concept and self-efficacy revisited. A few notable differences and important similarities. In W. H. Marsh, R. G. Craven, \& D. McInerney, International advances in self research (pp. 6789). Greenwich: Information Age Publishing. Stankov, L., Lee, J., Luo, W., \& Hogan, D. J.
(2012). Confidence: A better predictor of academic achievement than self-efficacy, selfconcept and anxiety? Learning and Individual Differences, 22(6), 747-758.

Stocker, J., \& Faria, L. (2012). Competência percebida no ensino secundário: Do conceito à avaliação através de um questionário compósito. Psicologia, 26(2), 113-140.

Strong, W. B., Malina, R. M., Blimkie, C. J. R., Daniels, S. R., Dishman, R. K., Gutin, B., \& Trudeau, F. (2005). Evidence based physical activity for school-age youth. The Journal of Pediatrics, 146(6), 732-737.

Vagetti, G. C., Filho, B., C, V., Moreira, N. B., Oliveira, V., Mazzardo, O., \& Campos, W. (2014). Association between physical activity and quality of life in the elderly: A systematic review, 2000-2012. Revista Brasileira de Psiquiatria, 36(1), 76-88.

Valle, A., Núñez, J. C., Cabanach, R. G., González-Pienda, J. A., Rodriguez, S., Rosário, P., ... Cerezo, R. (2009). Academic goals and learning quality in higher education students. The Spanish Journal of Psychology, 12, 96-105.

Vaz Serra, A. (1988). O autoconceito. Análise Psicológica, 6(2), 101-110.

Veas, A., Castejón, J.-L., Gilar, R., \& Miñano, P. (2015). Academic achievement in early adolescence: The influence of cognitive and non-cognitive variables. The Journal of General Psychology, 142(4), 273-294.

Veiga, F. H. (2005). Novos elementos acerca da escala de autoconceito "Piers-Harris Children's Self-Concept Scale”. In E. C. Martins (Ed.), Actas do VIII Congresso da Sociedade Portuguesa de Ciências da Educação (pp. 1085-1091). Castelo Branco: Instituto Politécnico de Castelo Branco.

Veiga, F. H., \& Domingues, D. (2012). A escala Piers-Harris Children's Self-Concept Scale: Uma versão com respostas de um a seis. In L. Mata, F. Peixoto, J. Morgado, J. C. Silva, \& V. Monteiro (Eds.), Atas do $12^{\circ}$ Colóquio Psicologia e Educação - Educação, aprendizagem e desenvolvimento: Olhares 
contemporâneos através da investigação e da prática (pp. 223-238). Lisboa: ISPA.

Von Elm, E., Altman, D. G., Egger, M., Pocock, S. J., Gøtzsche, P. C., \& Vandenbroucke, J. P. (2014). The strengthening the reporting of observational studies in epidemiology (STROBE) statement: Guidelines for reporting observational studies. International Journal of Surgery, 12(12), 1495-1499.

Zimmerman, B. J. (2008). Investigating selfregulation and motivation: Historical background, methodological developments, and future prospects. American Educational Research Journal, 45(1), 166-183.
THE SELF-CONCEPT OF HIGH SCHOOL STUDENTS RELATED WITH ACADEMIC PERFORMANCE: A SYSTEMATIC REVIEW

\section{Abstract}

The aim of the study was to systematically review the studies that analyzed the relationship between self-concept and academic achievement in high school students, as well as to verify which variables and contexts within the school were approached together with self-concept, to better explain this relationship. The review included studies published in the last ten years in the scientific electronic databases: ERIC/ Thesaurus, MEDLINE/Pubmed, SportDiscus, SciELO and Web of Science. The descriptors that characterized the components of selfconcept used were those of self, self-concept and self-psychology, and the descriptors related to academic achievement, academic achievement, underachievement, educational achievement, perception of competence, competence perception and self-efficacy. Ten studies were included in the review. The relationship between self-concept and academic performance was consistent and confirmed in nine of the ten papers. Other variables that were important in explaining the academic achievement were academic motivation, the practice of extracurricular activities, academic self-efficacy and parental involvement.

KeY-WORDS: Students; Self-psychology; Adolescent; Performance assessment 
EL AUTOCONCEPTO DE ESTUDIANTES DE ENSEÑANZA MEDIA Y SU RELACIÓN CON EL DESEMPEÑO ACADÉMICO: UNA REVISIÓN SISTEMÁT ICA

\section{RESUMEN}

El objetivo del estudio fue revisar sistemáticamente las investigaciones que analizaron la relación del autoconcepto con el rendimiento académico de adolescentes de la enseñanza media, así como verificar qué variables y contextos dentro de la escuela fueron abordados en conjunto al autoconcepto, para explicar mejor esta relación. La revisión incluyó estudios publicados en los últimos diez años en las bases electrónicas de trabajos científicos: ERIC/ Thesaurus, MEDLINE/Pubmed, SportDiscus, SciELO y Web of Science. Se utilizaron los descriptores referentes al autoconcepto (self, self-concept y self-psychology) y los descriptores relacionados con el rendimiento académico, en que también se consideró la capacidad académica percibida (academic achievement, underachievement, educational achievement, perception of competence, competence perception, self-efficacy). Diez estudios se incluyeron en la revisión. La relación entre autoconcepto y desempeño académico se mostró consistente y se confirmó en nueve de los diez trabajos.
Otras variables se presentaron importantes en la explicación del rendimiento académico, entre las cuales la motivación académica, la práctica de actividades extracurriculares, la auto-eficacia académica y la participación de los familiares.

Palabras-Clave: Estudiantes; Psicología del self; Adolescente; Evaluación de rendimiento

\footnotetext{
' Programa de Pós-Graduação em Educação, Universidade Federal do Paraná (PPGE-UFPR) \& Instituto Federal de Educação, Ciência e Tecnologia (IFPR), Brasil. ORCID: 0000-0002-9712-7571

"I Programa de Pós-Graduação em Educação, Universidade Federal do Paraná (PPGE-UFPR), Brasil. ORCID: 0000-00029071-7886

${ }^{\text {III }}$ Faculdade de Artes do Paraná \& Programa de Pós-Graduação em Educação, Universidade Federal do Paraná (PPGEUFPR), Brasil. ORCID: 0000-0003-0704-1297

Iv Programa de Pós-Graduação em Educação, Universidade Federal do Paraná (PPGE-UFPR), Brasil. ORCID: 0000-00029132-0514

v Programa de Pós-Graduação em Educação, Universidade Federal do Paraná (PPGE-UFPR), Brasil. ORCID: 0000-00028709-8471
} 